\title{
Medical Diagnostic Systems using Ensembles of Neural SOFM Classifiers
}

\author{
Christodoulos I. Christodoulou ${ }^{1,2}$, Constantinos. S. Pattichis ${ }^{3}$ \\ ${ }^{1}$ Department of Electronic Engineering, Queen Mary and Westfield College, \\ University of London, Mile End Road, London El 4NS, U.K. \\ ${ }^{2}$ Cyprus Institute of Neurology and Genetics, P.O.Box 3462, 1683 Nicosia, Cyprus \\ ${ }^{3}$ Department of Computer Science, University of Cyprus, 75 Kailipoleos Str., \\ P.O.Box 578, 1678 Nicosia, Cyprus \\ email: $\{$ cschr2, pattichi\}@ucy.ac.cy
}

\begin{abstract}
A design for medical diagnostic systems composed of ensembles of neural self organizing feature map (SOFM) classifiers is presented. Each SOFM classifier was fed with a different feature set extracted from the raw data and their results were combined using (i) majority voting and (ii) a confidence measure derived from the SOFM which weighted the contribution of each feature set to the final classification result. The following two diagnostic systems were developed: (i) a decision support system for the assessment of electronyographic (EMG) signals, and (ii) a system for the characterisation of carotid plaques. The results in this work show that combining the classification results of multiple classifiers using as input multiple feature sets, in conjunction with the use of a confidence measure can improve the overall classification performance of the system.
\end{abstract}

\section{Introduction}

In the case of difficult pattern recognition problems, the combination of the outputs of multiple classifiers using for input multiple feature sets extracted from the raw data, can improve the overall classification performance. In the case of noisy or of a limited amount of data, different classifiers often provide different generalisations by realising different decision boundaries. Also, different feature sets provide different representations of the input patterns, containing different classification information. Selecting the best classifier or the best feature set is not necessarily the ideal choice, since potentially valuable information contained in the less successful feature sets or classifiers may not be taken into alccount. The combination of the results of the different features and the different classifiers increases the probability that the errors of the individual features or classifiers may be compensated by the correct results of the rest [1]. Furthermore according to Perrone [2] the performance of the combiner is never worse than the average of the individual classifiers, but not necessarily better than the best classifier. The use of a confidence measure by weighting the individual classification results before combining can further improve the overall performance.

In this work a design for a medical diagnostic system composed of ensembles of self organizing feature map
(SOIM) classifiers is presented. The classification results of multiple classifiers inputted with multiple feature sets were combined using (i) majority voting and (ii) a confidence measure derived from the SOFM. The design was used for the development of two diagnostic systems: (i) a decision support system for the assessment of the electromyographic (EMG) signals, and (ii) a computer aided system which facilitates the automated characterisation of carotid plaques. The objective of this study was to investigate the usefulness of combining neural network classifiers in the development of medical diagnostic systems.

\section{Method}

Following the multiple feature extraction from the raw data, feature classification was implemented using multiple SOFM classifiers.

\subsection{SOFM classifier with a confidence measure}

The SOFM was chosen because it is an unsupervised learning algorithm where the input patterns are freely distributed over the output node matrix [3]. The weights are adapted without supervision in such a way, so that the density distribution of the input data is preserved and represented on the output nodes. This mapping of similar input patterns to output nodes which are close to each other represents a discretisation of the input space, allowing a visualization of the distribution of the input data. The output nodes are usually ordered in a two dimensional grid, and at the end of the training phase, the output nodes are labelled with the class of the majority of the input patterns of the training set, assigned to cach node. In the evaluation phase, an input pattern is assigned to the output node with the weight vector closest to the input vector, and it is said to belong to the class label of the winning output node where it has been assigned.

The SOFM classifier yielded also a confidence measure on how reliable the classification result was. The confidence measure was calculated based on the classes of the nearest neighbours on the self organizing map. For this purpose the output nodes in a neighbourhood window centered at the winning node were considered. Several window sizes were tested. The SOFM classifier was first trained with the training set and each output node was labelled with the number of the training input 
patterns from each class assigned to it. In the evaluation phase a new input pattern was assigned to a winning output node. The number of training input patterns per class assigned to each node in the neighbourhood window around the winning node, were counted. The evaluation input pattern was classified to the class of the majority of the training input patterns. The confidence measure was calculated as the percentage of the majority of the training input patterns to the total number of the training input patterns in the neighbourhood window. More specific in order to set its range from 0 to $1(0=$ low confidence, 1 = high confidence) the confidence measure was calculated as

$$
\text { Conf }=2\left(\max \left\{S N_{1}, S N_{2}\right\} /\left(S N_{1}+S N_{2}\right)\right)-1
$$

where $S N_{m}$ is the number of the input patterns in the neighbourhood window for the $m$ possible classes:

$$
S N_{m}=\sum_{i=1}^{l} N_{m i}
$$

where $N_{m i}$ is the number of the training patterns of the class $m$ assigned to the output node $i$. $L$ is the number of the output nodes in the $R \times R$ window with $L=R^{2}$.

A further enhancement in the calculation of the confidence measure is to give to the output nodes nearest to the winning output node a greater weight than the ones farther away. So a windowing mask is used and the $N_{m i}$ is multiplied with the value of the mask depending on the distance of the output node from the winning output node. In this case Eq. 2 is modified into

$$
S N_{m}=\sum_{i=1}^{l} N_{m i} W_{i}
$$

where $W_{i}$ is the weight value at the output node $i$. The winning output node is given weight value equal to 1 whereas the rest weight values of the windowing mask are decreasing exponential with the distance from the winning output node.

\subsection{Combining classifiers with a confidence measure} In this work, the usefulness of combining neural network classifiers was investigated for the development of medical diagnostic systems. Different feature sets were extracted from the raw data and were inputted into the SOFM classifiers. The different classification results were combined using (i) majority voting where the input pattern was assigned to the class with the greater number of votes, and (ii) with the use of the confidence measure where the final classification result was the average of the different confidence measures. The confidence measure was computed as described in section 2.1 and decided the contribution of each feature set to the final result. The idea is that some feature sets may be more successful for specific regions of the input population.

\section{Applications}

The following two medical diagnostic systems were developed using the methodology described above:

(i) A decision support system for the assessment of the electromyographic (EMG) signals recorded from normal subjects and subjects suffering with myopathy and motor neuron disease.

(ii) A computer aided system which facilitates the automated characterisation of carotid plaques recorded from high resolution ultrasound images for the identification of individuals with asymptomatic carotid stenosis at risk of stroke.

\subsection{The EMG Decision Support System}

The diagnosis of neuromuscular disorders is a naturally complex and difficult problem to investigate. This group of disorders affects the brain and spinal cord, nerves, or muscles. The main characteristic of this group of disorders is muscular weakness and/or wasting, with the life expectancy of many sufferers being considerably reduced. Early detection and diagnosis of these diseases by laboratory tests and neurophysiological examinations, like EMG are essential for their prognosis, management, and prevention. The shapes of motor unit action potentials (MUAPs) composing the EMG signal, provide an important source of information for the assessment of neuromuscular disorders.

In this study a total of 800 MUAPs were recorded from 40 subjects, 12 normal (NOR), 13 suffering from myopathy (MYO) and 15 from motor neuron disease (MND). The neural network system was trained and tested for the three classes. Ten different bootstrap sets with eight subjects from each group were randomly selected to form the ANN training set, whereas the remaining subjects formed the ANN evaluation set [4].

For each subject a matrix of 20 MUAP waveforms $\times 512$ samples, was available for further processing. From each MUAP waveform the following feature sets were extracted [4]:

\section{(i) Time domain parameters}

The following time domain parameters were computed from the MUAP waveforms: duration, spike duration, amplitude, area, spike area, number of phases and number of turns.

\section{(ii) Frequency domain parameters}

The following frequency domain parameters were computed from the MUAP waveforms: spectral moments of order 0,1 and $2\left(M_{0}, M_{3}\right.$, and $\left.M_{2}\right)$, median frequency and quality factor.

\section{(iii) Cepstral coefficients}

The cepstral coefficicnts $c_{1}$ to $c_{12}$ were computed from the MUAP signal.

\section{(iv) Wavelet coefficients}

The wavelet transform provides a linear two dimensional time-frequency representation and was investigated for describing MUAP morphology. Three different wavelets were computed: Daubechies 4 (DAU4) with four coefficients, Chui $(C H)$ and Battle-Lemarie $(B L)$.

For each subject, the average vector of 20 MUAPs for each feature set was computed which was subsequently used as input to the SOFM classifiers. 
3.2 Computer aided classification of carotid plaques There are indications that the morphology of atherosclerotic carotid plaques, obtained by high resolution ultrasound imaging, have prognostic implications [5]. Smooth surface, echogenicity and a homogenous texture are characteristics of stable plaques, whereas irregular surface, echolucency and a heterogenous texture are characteristics of potentially unstable plaques. The objective of this work was to develop a computer aided system which will facilitate the automated characterisation of carotid plaques recorded from high resolution ultrasound images (duplex scanning and colour flow imaging). This work is part of an European Union project (Biomed 2 Program - PL 950629) carried out in centers all over Europe and coordinated by the St. Mary's Hospital [6]. The developed system should be able, based on extracted texture feature and shape parameters, to automatically classify plaques into one of the following types: (i) Symptomatic because of ipsilateral hemispheric symptoms and (ii) Asymptomatic because they were not connected with ipsilateral hemispheric events. The aim is to identify patients at risk of stroke.

The plaque segments were outlined from the uitrasound images manually by the expert physician. A total of 166 carotid plaque images (76 symptomatic +90 asymptomatic) has been processed. For training the system 58 symptomatic and 58 asymptomatic plaques were used whereas for evaluation of the system the remaining 18 symptomatic and 32 asymptomatic plaques were used. The system was trained and evaluated using five different bootstrap sets where in each set 116 different plaques were selected at random for training and 50 different plaques for evaluation. Texture features and shape parameters were extracted from the segmented plaque images in order to be used for the classification of the carotid plaques. Texture refers to the spatial interrelationships and arrangement of the basic elements of an image [7]. Visually, these spatial interrelationships and arrangements of the image pixels are seen as variations in the intensity patterns or gray tones. Therefore texture features have to be derived from the gray tones of the image.

In this study, 10 different texture feature sets (a total number of 61 features) were extracted from the plaque segments and used as input to the SOFM classifiers, using the following algorithms:

\section{(i) First Order Statistics (FOS),}

1) Mean value, 2) Median value, 3) Standard Deviation, 4) Skewness, 5) Kurtosis.

\section{(ii) Spatial Gray Level Dependence Matrices} (SGLDM), [8]

1) Angular second moment, 2) Contrast, 3) Correlation, 4) Sum of squares: variance, 5) Inverse difference moment, 6) Sum average, 7) Sum variance, 8) Sum entropy, 9) Entropy, 10) Difference variance, 11)
Difference entropy, 12), 13) Information measures of cortrelation.

For each feature the mean values and the range of values were computed and used as two different feature sets.

(iii) Gray Level Difference Statistics (GLDS), [9]

1) Contrast, 2) Angular second moment, 3) Entropy, 4) Mean.

(iv) Neighbourhood Gray Tone Difference Matrix (NGTDM), [7]

1) Coarseness, 2) Contrast, 3) Business, 4) Complexity, 5) Strength.

(v) Statistical Feature Matrix (SFM), [10]

1) Coarseness, 2) Contrast, 3) Periodicity, 4) Roughness. (vi) Laws Texture Energy Measures (TEM), [10|

1) LL - texture energy from LL kernel, 2) EE - texture energy from EE kernel, 3) SS - texture energy from SS kernel, 4) LE - average texture energy from LE and EL kernels, 5) ES - average texture energy from ES and SE kernels, 6) LS - average texture energy from LS and SL kernels.

(vii) Fractal Dimension Texture Analysis (FDTA), [10] $H^{(k)}$ parameter (Hurst coeff.) for resolutions $k=1,2,3,4$. (viii) Fourier Power Spectrum (FPS), [10]

1) Radial sum, 2) Angular sum.

(ix) Shape Parameters

1) X - coord. maximum length, 2) $Y$ - coord. maximum length, 3) Area, 4) Pcrimeter, 5) Perimeter ${ }^{2} /$ Area.

Figure 1 illustrates the distribution of the 166 carotid plaques on a $12 \times 12$ SOFM using as input all the 61 features.

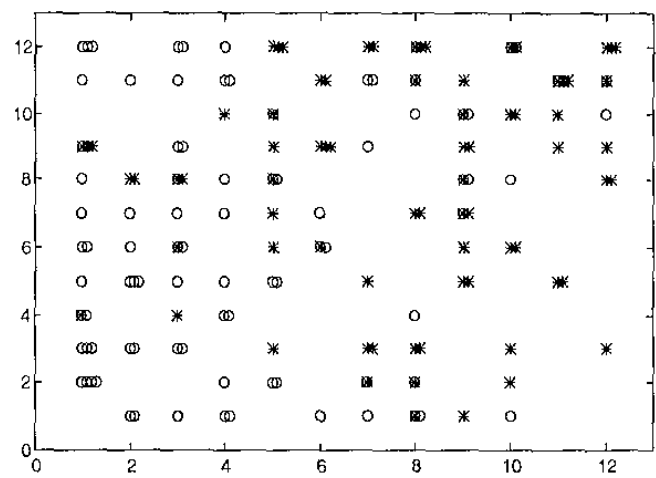

Fig. 1 Distribution of 166 carotid placues (76 Symptomatic + 90 Asymptomatic) on a $12 \times 12$ SOFM using as input all the 61 leatures $(*=$ Symptomatic, $0=\Lambda$ symptomatic ).

\section{Results}

For the EMG classification system the SOFM classifiers were trained for 3000 learning epochs and the 24 subjects of the training set were distributed over a $6 \times 6$ output node matrix. For the carotid plaque classification system the SOFM classifiers were trained for 5000 learning epochs and a $12 \times 12$ output node architecture was used. For the several neighbourhood windows used the $3 \times 3$ was found to give overall best results. In this window size the 8 output nodes around the winning 
output node were considered as described in section 2.1. Tables I and II tabulate the results for the two diagnostic systems respectively. In both systems, the diagnostic yield when combining with majority voting exceeded the average diagnostic yield of the individual feature sets. Combining based on a confidence measure improved furthermore the diagnostic yield. For the EMG classification system dominant best feature set was the time domain parameters with $79.2 \%$ which was almost equal to the diagnostic yield $79.6 \%$ when combining with the confidence measure. In the carotid plaque classification system best feature set was the GLDS with $68.8 \%$ which was significantly lower than the diagnostic yield when combining with the confidence measure which was $76.4 \%$.

Table I Diagnostic yield of the EMG diagnostic system, alter bootstrapping the available data for ten different sets of subjects.

\begin{tabular}{|c|c|c|c|}
\hline $\mathrm{Nr}$ & Feature set & $\begin{array}{c}\text { Vector } \\
\text { size }\end{array}$ & $\begin{array}{c}\text { Diagnostic } \\
\text { yield \% }\end{array}$ \\
\hline 1 & Time domain parameters & 7 & $79.2 \pm 11.2$ \\
\hline 2 & Frequency domain param. & 5 & $70.6 \pm 14.9$ \\
\hline 3 & Cepstral coefficients & 12 & $67.3 \pm 10.3$ \\
\hline 4 & Wavelet transform DAU4 & 16 & $12.7 \pm 9.2$ \\
\hline 5 & Wavelet transform CH & 16 & $58.8 \pm 11.0$ \\
\hline 6 & Wavelet transform BL & 16 & $66.2 \pm 8.9$ \\
\hline \multicolumn{3}{r}{ Average: } & $69.1 \pm 11.0$ \\
\hline \multicolumn{3}{|c|}{ Combining with majority voting: } & $76.9 \pm 7.5$ \\
\hline Combining with confidence measure: & $79.6 \pm 6.9$ \\
\hline
\end{tabular}

Table II Diagnostic yicld of the carotid plaque diagnostic system, after bootstrapping the available data lor five different sets of plaques.

\begin{tabular}{|c|c|c|c|}
\hline $\mathrm{Nr}$ & Feature set & $\begin{array}{c}\text { Vector } \\
\text { size }\end{array}$ & $\begin{array}{c}\text { Diagnostic } \\
\text { yield } \%\end{array}$ \\
\hline 1 & FOS & 5 & $68.4 \pm 7.1$ \\
\hline 2 & SGLDM (mean) & 13 & $65.2 \pm 6.0$ \\
\hline 3 & SGLDM (range) & 13 & $62.8 \pm 8.5$ \\
\hline 4 & GLDS & 4 & $68.8 \pm 7.1$ \\
\hline 5 & NGTDM & 5 & $61.2 \pm 4.3$ \\
\hline 6 & SFM & 4 & $63.2 \pm 4.1$ \\
\hline 7 & TEM & 6 & $66.0 \pm 4.6$ \\
\hline 8 & FDT & 4 & $63.6 \pm 7.1$ \\
\hline 9 & FPS & 2 & $53.6 \pm 4.3$ \\
\hline 10 & Shape parameters & 5 & $51.6 \pm 6.7$ \\
\hline \multicolumn{3}{|c|}{ Average : } & $62.4 \pm 6.0$ \\
\hline \multicolumn{3}{|c|}{ Combining with majority voting: } & $70.4 \pm 7.7$ \\
\hline Combining with confidence measure: & $76.4 \pm 0.0$ \\
\hline
\end{tabular}

\section{Conclusions}

The results in this work show that modular neural networks are a promising tool in solving difficult pattern recognition problems. The combining of multiple neural classifiers using as input multiple feature sets, in conjunction with the use of a confidence measure for weighting the classification results, can significantly improve the overall classification performance. The performance improvement is more obvious when no dominant best feature set is available, as this was the case with the carotid plaque classification system. The choice of the SOFM classifier is well suited when the characteristics of the different classes overlap which was the case in the two systems described. In such cases the unsupervised learning SOFM algorithm results in an efficient mapping of the training data and leads to a satisfactory classification performance, whereas supervised learning algorithms like the backpropagation and the radial basis function algorithms fail to converge. The diagnostic results could be verified further using more data from more subjects, both for training and evaluating the systems.

\section{Acknowledgements}

This work was partly supported by the Cyprus Planning Bureau, the Cyprus Institute of Neurology and Genctics and the University of Cyprus.

\section{References}

[1] Tumer K., Ghosh J., "Theortical foundations of linear and order statistics combiners for neural pattern classifiers", Dept. of Electrical and Computer Engineering, University of Texas, Austin, 1996.

[2] Perrone M.P., "Averaging/Modular Techniques for Neural Networks", The Ilandbook of Brain Theory and Neural Networks, ed. by M.A. Arbib, MIT Press, Cambridge, Massachusctts, pp. 126-129, 1995.

13] Kohonen T., "The Self-Organizing Map", Proceedings of the IFEE, Vol. 78, No. 9, pp. 1464-1480, Scpt. 1990.

[4] Christodoulou C.I., Pattichis C.S., Fincham W.F., "A Modular Neural Network Decision Support System in EMG Diagnosis", Journal of Intelligent Systems, Special issue on Computational Intelligent Diagnostic Systems in Medicine, ed. by C.N. Schizas, Volume 8, Nos. 1-2, pp. 99-143, 1998.

[5] JEMU: Consensus concerning the morphology and the risk of carotid plaques, Vol. 176, Paris 14 December 1996.

[6] ACSRS: The value of noninvasive investigations in the identification of inclividuals with Asymplomatic Carotid Stenosis at Risk of Stroke, Protocol of Bioned 2 Program - Pl, 950629, 1995.

[7] Amadasun M., King. R., "Textural Features Corresponding to Textural Properties", IEEE Transactions on Systems, Man, and Cybernetics, Vol. 19, No 5, Sept./Oct. 1989.

[8] Haralick R.M., Shanmugam K., Dinstein I., "Texture Features for Image Classification", IFEE Transactions on Systems, Man., and Cybernetics, Vol. SMC-3, pp. 610621, Nov. 1973.

19] Weszka J.S., Dyer C.R., Rosenfield A., "A Comparative Study of Texture Measures for Terrain Classification", IEEE Transactions on Systems, Man. \& Cybernetics, Vol. SMC-6, April 1976.

[10] Wu Chung-Ming, Chen Yung-Chang, Hsich Kai-Sheng, "Texture Features for Classification of Ultrasonic Liver Images", IEEE Transactions on Medical Inaging, Vol. I I, No 3, June 1992. 